\title{
THESIS
}

$378.7 \mathrm{MI}$

$x \times 523$

UMLD 

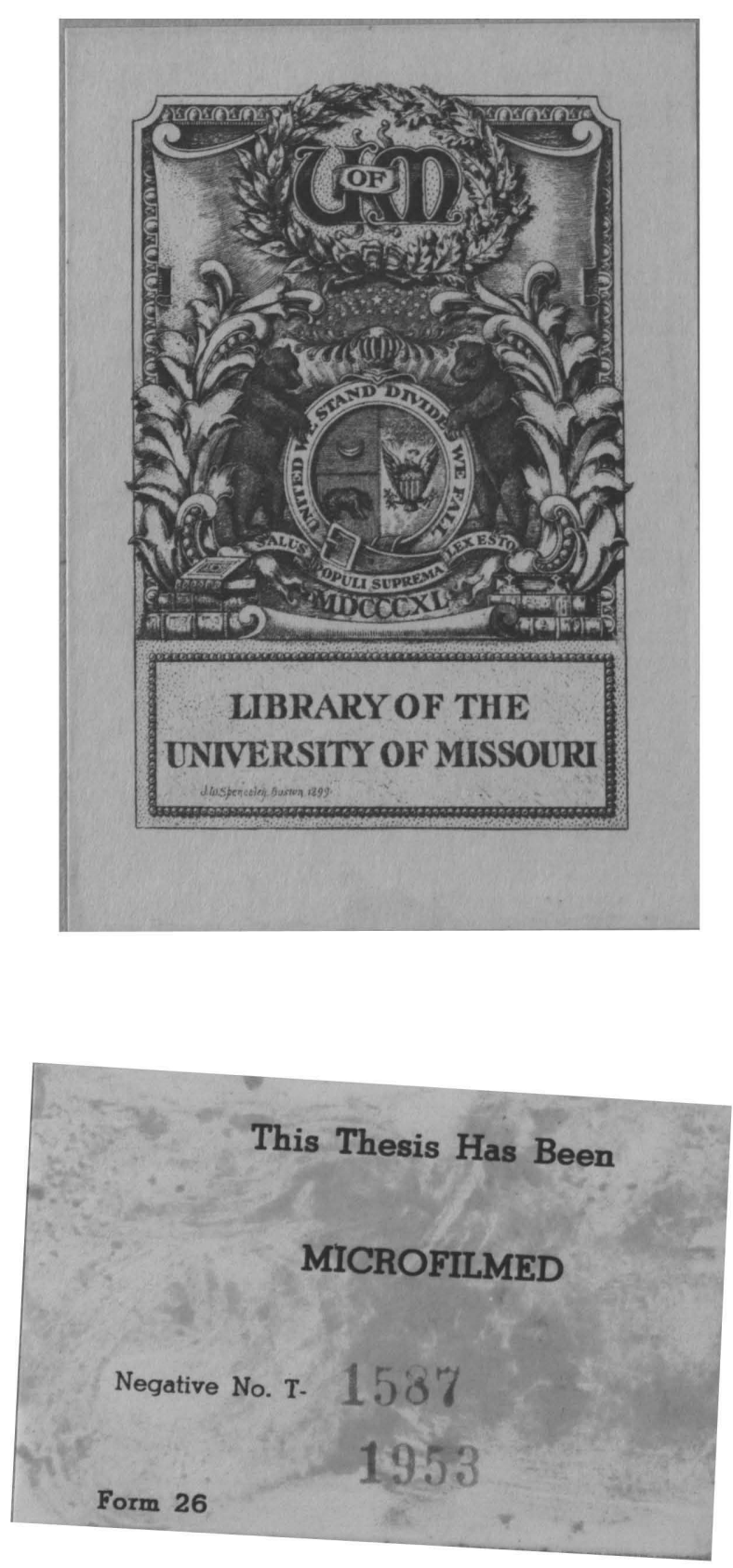






\section{STUDY OF THE CONVERGENCE OF SERIES IN \\ CERTAIN ORTHOGONAI FUNCTIONS}

$$
\text { by }
$$

Reuben Axel Wester, A. B.

\section{SUBMITTED IN PARTIAI FUIFIIIMENT OF THE REQUIREMENTS FOR THE DEGREE OF MASTER OF ARTS in the}

GRADUATE SCHOOI.

$$
\text { of the }
$$

UNIVERSITY OF MISSOURI 



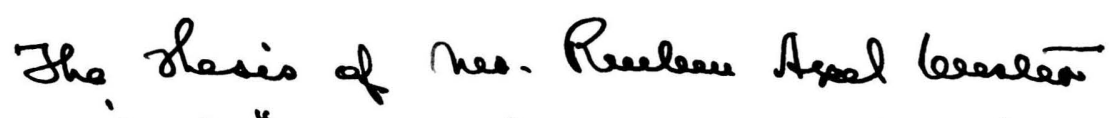
entateed "sters of the senerapques of renies is ceatain orikagonal fanction" serleniteed in pachial frefiel ment of to requinements for the degare of hraster of chets, har baw exerittem reuder ver sireation and nesets eviet nem approual.

QD blelooge Depardment freach weats is . vecuersiely of heissoenci near $13,1914$. 





\section{B1bliography.}

1. Welerstrass, "Über die analytische Darstellbarke1t sogenannter willkürlicher Funotionen einer reolen Veränderlichen." Sitzungsberlohte der Kgl. Preuss. Akademie der Wissenschaften, 1885, Pages 633 - 639, 789 - .

2. Lobesgue, "Sk̂r la représentation approchéo des fonotions". Rendicont1 del Ciroolo Matematico di Palermo, Vol. XXVI, 1908, Pages 325 -328.

3. de la Vallée Poussin, "Sŭr l'approximation des fonctions d'une variable réele et de lours derivées par des polynômes et des sultes limitées de Fourier". Bulletins de l'Acodemie royale de Belgique, Classe des Solences, 1908, Pages 193 - 254.

4. de la Vallée Poussin, "Ilote sur l'approximation par un polynôme d'une fonction dont la derivée est a variation bornée". Bulletins de l'Aoademie royale de Belgique, Classe des Solences, 1908, Pages 403 -410.

6. Iebesgue, "Sux la representation trigonometrique approchée des fonctions satisfaisant $\grave{a}$ une condition de lipschitz". Bulletin de la Soolete Math. de France, Vol. XXXVIII, 1910, Pages 184 - 210.

5. Lebesgue, "Sur les integrales singulieres". Annales de la Faculte de Toulouse, Series 3, Vol. I, 1910, pages 25 - 117.

7. Jackson, "Über die Genaugigkeit der Annäherung stetiger Funktionen durch ganse rationale Funktionen gegebenen Grades und trigonometrisohe Summen gegebener Ordnung". InauguralDissertation; Gottingen, 1911. In the proface of this dissertation Jackson gives a verg extens1ve bibliography on the subjeot of epproximations by means of polynomials 

and trigonometrio sums.

8. Jackson, "On the approximate representation of an indefinite integral and the degree of convergence of related Fourier's series". Trans. Amer. Math. Soc. Vol. XIV, 1913, pages 343 - 364 .

9. Jackson, "On approximations by trigonometric sums and polynomials". Trans. Amer. Math. Soc. Vol. XII, 1912, pages 491 - 517 .

10. Jackson, "On the degree of convergence of the development of a continuous function according to Iegendre's polynomials". Trans. Amer. Math. Soc. Vol. XII, 1912.

11. Gronwall, "On the degree of convergence of a Iaplace's series". Trans. Amer. Math. Soc. Vol.XV, 1914.

12. Stieltjes, "Table des valeurs des sommes $s_{k}=\sum_{1}^{\infty} n^{-k}$. Acta Mathematica, Vol. X, pages 299 - 302.

13. Byerly, "An elementary treatise on Fourier's series and Spherical, cylindrical and ellipsoidal harmonics".

14. Whittaker, "Modern Analysis".

15. Riemann-Weber, "Die partiellen differential Gleichungen der mathematischen Physik. Page 185, Vol. I. 

Introduction.

The question of the accuracy with which a given function, say $f(x)$, can be expressed by means of a known function or in a series of known functions is a very important one. Such developements are continually arising in problems in applied mathematics such as in mathematical physics and astronomy. The value of ang developement depends largely on the accuracy with which one can judge its error at any point.

Reviewing hastily some of the work that has been done along this line, we see that Weierstrass ${ }^{l}$ was the first to give the proof for the following theorem:

If in the interval $a \leqq x \leqq b \quad f(x)$ is a single valued, defined, continuous and real function of the real variable $x$, and if $\delta$ is an arbitrarily chosen positive quantity, then there is an integral rational function $P(x)$ such that throughout the interval $a \leq x \leqq b, \quad|P(x)-P(x)|<\delta$.

It is clear that in general the degree of the polynomial $P(x)$ is dependent upon $\delta$. As $\delta$ is chosen smaller and smaller the degree of $\mathrm{P}(\mathrm{x})$ will in general increase. Iesbesgue ${ }^{2}$ has cosidered the question of what the relation is between $\delta$ and the degree of the polynomial of lowest degree which satisfies the above inequality throughout the interval for any given function, $f(x)$.

For any polynomial $P(x)$ of the nth degree, the expression $|f(x)-P(x)|$ has an upper limit in the interval $a \leqq x \leqq b$. This upper limit is a function of the coefticients of $P(x)$. If we consider several polynomials of the $n^{\text {th }}$ degree or lower, this function has a definite lower limit which for a given a, b, and 

$f(x)$ depeñas only on $n$. Iet us eall this lover limit $\boldsymbol{\varphi ( n )}$. It is in general positive. It becones zero only when $f(x)$ is loself a polynomid of the $\mathrm{n}^{\text {th }}$ aecreo or lover. The theorem by Weierstrass states that for a continuous function $f(x)$, the $\lim _{n=\infty} \Phi(n)=0$. Introducing the notation $\varphi(n)=0(\psi(n))$ and $\varphi(n)=o(\psi(n))$, by which we shall mean that for $n$ sufficientIf large $|\varphi(n)|<A \psi(n)$, where $A$ is a constant independent of $n$, or that $\lim _{n=\infty} f(n) / \psi(n)=0$ respectively, we can state Weierstrass' result by saying that $\varphi(n)=o(I)$ for a continuous function. Iesbesgue ${ }^{2}$ gives a result which says that in case a funotion satisfies a Iipschitz condition, i.e.

$$
\left|f\left(x_{2}\right)-f\left(x_{1}\right)\right| \leqq \lambda\left|x_{2}-x_{1}\right| \text {, }
$$

where $\lambda$ is a constant, $\varphi(n)=0\left(\sqrt{\frac{\log n}{n}}\right)$.

Assuming practically the same conditions de la Vallee Poussin ${ }^{3}$ finds that $\varphi(n)=0\left(\frac{1}{\sqrt{n}}\right)$. Iater ${ }^{4}$ he finds that when $f(x)$ possesses a derivative of limited variation, $\varphi(n)=O(\underline{l})$. Iesbesgue ${ }^{5}$ has considered the case where $f(x)$ satisfies a Iipschitz-Dini condition, i.e. $\lim \delta=0(\delta) \log \delta=0$ where $\omega(\delta)$ represents the upper limit of the difference between the maximum and minimum values assumed by $f(x)$ in an interval of length $\delta$. He finds that in such a case $\varphi(n)=0\left(\frac{1}{\log n}\right)$.

More in line with this paper is the work done on approximations by means of trigonometric sums. There is a very close relation between trigonometric and polynomial approximations. From a result by Iesbesgue ${ }^{6}$ about the convergence of $a$ Fourier's series one can get, assuming the Iifschitz condition, that $\varphi(n)=0\left(\frac{\log n}{n}\right)$. Jackson ${ }^{7}$ gives a somewhat better result finding that $\varphi(n)=O\left(\frac{l}{n}\right)$. He also shows that there exist func- 

tions satisfying a Iipschitz condition for which $\varphi(n)$ is not $o(\underline{l})$. In the case of trigonometric approximations we will write $\tau(n)$ in place of $\varphi(n)$ to distinguish the two cases. Iesbesgue 5 has found that in case a function satisfies a Iipschitz condition $\tau(n)=O\left(\frac{l}{n}\right)$ and later ${ }^{6}$ he finds that $\tau(n)=O\left(\frac{\log n}{n}\right)$. Jackson's result is that $\tau(n)=O\left(\frac{l}{n}\right)$. In a later paper ${ }^{9}$ he proves the following theorem:

"Theorem I. There exists an absolute numerical constant $\mathrm{K}$ having the following property: If $f(x)$ is a real function of the real variable $x$, of period $2 \pi$, which everywhere satisfies the Iipschitz condition $\left|f\left(x_{2}\right)-f\left(x_{1}\right)\right| \leqq \lambda\left|x_{2}-x_{1}\right|$,

$\lambda$ being a constant, then there exists for every positive integral value of $n$ a trigonometric sum $T_{n}(x)$, of the $n^{\text {th }}$ order at the most, such that for all values of $x \quad\left|f(x)-T_{n}(x)\right| \leqq \frac{K \lambda}{n}$." He finds that $\frac{\pi}{2}<K \leqq 3$. A generalization of this theorem is his Theorem III of, the same paper. "

"For each positive integral value of $\mathrm{k}$ there exists a constant $K_{k}$ having the following property: If $f(x)$ is a function of period $2 \pi$ possessing a $(k-1)^{\text {th }}$ derivative which everywhere satisfies the Iipschitz condition $\mid f\left(x_{2}-f\left(x_{1}\right)|\leqq \lambda| x_{2}-x_{1} \mid\right.$

$\lambda$ being a constant, then there exist for every positive integral value of $n$ a trigonometric sum $T_{n}(x)$, of the $n^{\text {th }}$ order at most, such that for all values of $x$

$$
\left|f(x)-T_{n}(x)\right| \leqq \frac{K_{k} \lambda}{n^{k}} \cdot "
$$

Similar theorems are given for polynomial approximations.

Jackson has also given results for the degree of convergence of certain series. For instance in the case of a Fourier's series he gets, in the paper just quoted, the follow- 

ing theorem:

"Theorem $X$. If $f(x)$ is a function of period $2 \pi$ satisfying the Iipschitz condition, it is represented by the partial sum of its Fourier's series to terms of the $n^{\text {th }}$ order, provided $n \geq 5$, with an error not exceeding $6 \lambda(\underline{\log n}) . "$

In a later paper ${ }^{8}$ he has a generalization of this result, viz.,

"Theorem X. If $f(x)$ is a function of period $2 \pi$ possessing a $(\mathrm{k}-1)^{\text {th }}$ derivative whioh everywhere satisfies the Iipsohitz conaition $\left.\mid f^{k-7}\left(x_{2}\right)-e^{k-7} x_{1}\right)|\leqq \lambda| x_{2}-x_{1} \mid$, where $\lambda$ is a constant, then $f(x)$ is everywhere approximately represented by the partial sum of its Fourier's series to terms of the $n^{\text {th }}$

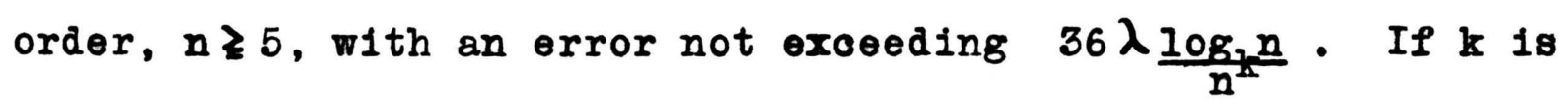
odd, the faotor 36 may be replaced by 12."

For the case of the developement of a function in series of Iegendre's polynomials Jackson ${ }^{10}$ has also given a result. Denoting by $r_{n}(x)$ the difference $\sum_{m=0}^{\infty} a_{m} P_{m}(x)-f(x)$, where $P_{m}(x)$ are the polynomials of Iegendre, and $a_{m}=\frac{2 m+1}{2} \int_{-1}^{+l} f(x) P_{m}(x) d x$ he gets:

"Theorem II. If $f(x)$ is a function possessing a $(k-1)^{\text {th }}$ derivative whioh satisfies a Iipschitz condition throughout the closed interval $(1,-1)$, then, for this function $f(x)$.

$$
\left|r_{n}(x)\right|=0\left(\frac{\log n}{n^{K}}\right) . "
$$

Gronwall ${ }^{11}$ gives a somewhat more general result in discussing the degree of convergence of a Iaplace's series of which a Iegendre's series is but a special case.

In thie present paper we will develope some theorems concerning the degree of convergence of certain series, in 

partioular a Fourier's series, a Iogendre's series, and a series of Bessel's functions, getting results which are indeed very much similar to the ones already mentioned but which are derived in a much simpler manner. Before proceeding directly to the proof of these theorems, however, we will give some theorems showing the conditions under which the Fourier coefficients in the developement of a function will converge to zero. 

Chapter I.

1. Total variation finite. Iet $f(x)$ be a real function of the real variable $x$ and let us impose the condition that the total variation of $f(x)$ shall remain finite in an interval from $x=0$ to $x=2 \pi$. This condition forces $f(x)$ to be bounded in the interval under consideration $1 . e .$, it is possible to find a positive quantity $\mathbb{N}$ such that $|f(x)| \leqq \mathbb{N}$ when $0 \leqq x \leqq 2 \pi$.

We have then the Fourier developement:

$$
\begin{aligned}
f(x)=b_{0} & +b_{1} \cos x+b_{2} \cos 2 x+b_{3} \cos 3 x+\ldots-\ldots \\
& +a_{1} \sin x+a_{2} \sin 2 x+a_{3} \sin 3 x+\ldots-
\end{aligned}
$$

where $b_{k}=\frac{1}{\pi} \int_{0}^{2 \pi} f(x) \cos k x d x$, and $a_{k}=\frac{1}{\pi} \int_{0}^{2 \pi} f(x) \sin k x d x$.

Since the absolute value of the sin $k x$ and $\cos k x$ can never exceed unity we can study the degree of convergence of this developement by studying the degree of convergence of the coefficients, $b_{k}$ and $a_{k}$. These coefficients may indeed approach zero without the series converging but we shall study this convergence to zero of the coefficients before imposing further conditions.

We can write:

(2)

$$
\begin{aligned}
& b_{k}=\frac{1}{\pi} \int_{0}^{2 \pi} f(x) \cos k x d x=\frac{1}{\pi}\left\{\int_{0}^{\frac{\pi}{2 k}} f(x) \cos k x d x+\int_{\frac{\pi}{2 k}}^{\pi / k} f(x) \cos k x d x\right] \\
& \left.+\int_{\frac{\pi}{k}}^{\frac{3 \pi}{2 k}} f(x) \cos k x d x+--+\int_{\left(\frac{2 k-1}{k}\right) \pi}^{2 \pi} f(x) \cos k x d x+\int_{\left(\frac{4 k-1}{2 k}\right) \pi}^{\left(\frac{k}{k}\right) \pi} f(x) \cos k x d x\right\}
\end{aligned}
$$

And similarly: 

(3)

$$
a_{k}=\frac{1}{\pi} \int_{0}^{2 \pi} f(x) \sin k x d x=\frac{1}{\pi}\left\{\int_{0}^{\frac{\pi}{2 k}} f(x) \sin k x d x+\int_{\frac{\pi}{2 k}}^{\frac{\pi}{k}} f(x) \sin k x d x\right.
$$

$$
\left.+\int_{\frac{\pi}{k}}^{\frac{3 \pi}{2 k}} f(x) \sin k x d x+-++\int_{\left(\frac{2 k-1}{k}\right) \pi}^{\left(\frac{4 k-1}{2 k}\right) \pi} f(x) \sin k x d x+\int_{\left(\frac{4 k-1}{2 k}\right) \pi}^{2 \pi} f(x) \sin k x d x\right\}
$$

By the law of the mean we know that

$$
\int_{a}^{b} f(x) \varphi(x) d x=\mu \int_{a}^{b} \varphi(x) d x
$$

where $m<\mu<\mathbb{M}$, $m$ and $\mathbb{M}$ being the minimum and maximum values assumed by the function, $f(x)$, in the interval $a \leqq x \leqq b$. There is no restriction on $f(x)$ other than that it be bounded.

\section{Also we have that}

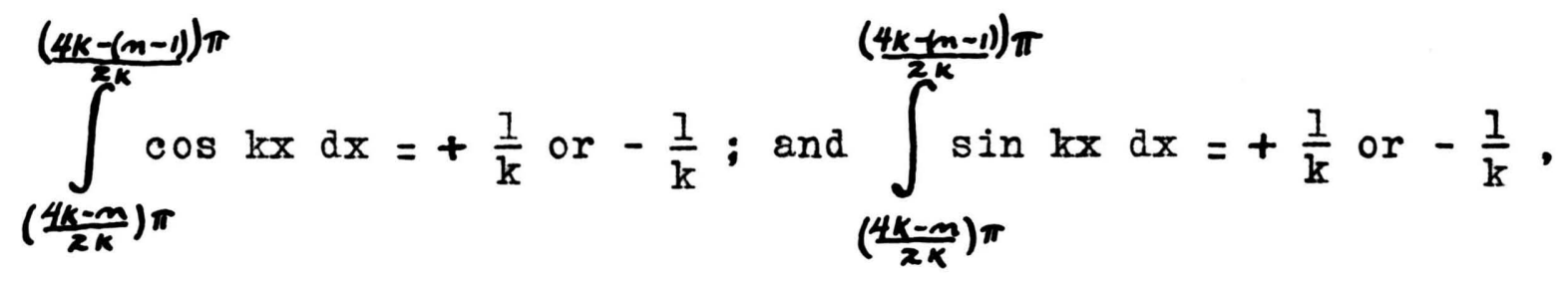

where $n=1,2,3, \ldots-\ldots \mathrm{k}$.

We will then have, after substitution in (2),

$$
b_{k}=\frac{1}{\pi}\left\{\frac{1}{k}\left(\mu_{1}-\mu_{2}-\mu_{3}+\mu_{4}+\mu_{5} \cdots-\mu_{4 k-2}-\mu_{4 k-1}+\mu_{4 k}\right)\right\} \text {, }
$$

where $\mu_{1}, \mu_{2}, \mu_{3}, \mu_{1},--$ etc., lie between the minimum and maximum values assumed by $f(x)$ in the intervals

$$
0 \leqq x \leqq \frac{\pi}{2 k}, \frac{\pi}{2 k} \leqq x \leqq \frac{\pi}{k}, \frac{\pi}{k} \leqq x \leqq \frac{3 \pi}{2 k},-\cdots \text { respectively. }
$$

Then $\left|b_{k}\right| \leqq\left\{\frac{1}{\pi}\left[\frac{1}{k}\left(\left|\mu_{1}-\mu_{2}\right|+\left|\mu_{4}-\mu_{3}\right|+\left|\mu_{5}-\mu_{6}\right|+\cdots+\left|\mu_{4 k}-\mu_{4 k-1}\right|\right)\right]\right\}$ (5)

Similarly after substitution in (3) we have, 



$$
a_{k}=\frac{1}{\pi}\left\{\frac{1}{k}\left(\mu_{0}+\mu_{1}-\mu_{z}-\mu_{1}+\mu_{4}+\mu_{5} \cdots+\mu_{4 k-3}-\mu_{4 k-2}-\mu_{4 k-1}\right)\right\} .
$$

where $\mu_{0}, \mu_{1}, \mu_{2}, \mu_{3}, \ldots-$ otc., lie between the minimum and and maximum values assumed by $f(x)$ in the intervals

$$
0 \leqq x \leqq \frac{\pi}{2 k}, \frac{\pi}{2 k} \leqq x \leqq \frac{\pi}{k}, \frac{\pi}{k} \leqq x \leqq \frac{3 \pi}{2 k},-- \text { respectively. }
$$

Then $\left|a_{k}\right| \leqq \frac{1}{\pi}\left\{\frac{1}{k}\left[\left|\mu_{0}-\mu_{4 k-1}\right|+\left|\mu_{1}-\mu_{2}\right|+\left|\mu_{4}-\mu_{3}\right|+\cdots+\left|\mu_{4 k-5}-\mu_{4 k-2}\right|\right]\right\}$

But in equation (5) each of the quantities $\left|\mu_{1}-\mu_{2}\right|$. $\left|\mu_{4}-\mu_{3}\right|,\left|\mu_{3}-\mu_{6}\right|,-$ - is evidently less than the total variation of $f(x)$ in the intervals

$$
0 \leqq x \leqq \frac{\pi}{k}, \frac{\pi}{k} \leqq x \leqq \frac{2 \pi}{k}, \frac{2 \pi}{k} \leqq x \leqq \frac{3 \pi}{k},- \text { respectively. }
$$

For denoting the total variation by $\omega$, we have $\omega_{1} \geqq \mathbb{M}_{1}-m_{1}$, where $M_{1}$ and $m_{1}$ are the maximum and minimum values of $f(x)$ in the interval $0 \leqq x \leqq \pi / k$. But $\mu_{1}>m_{1}$ and $\mu_{2}<M_{1}$, since the minimum of a function in an interval is always less than or at the most equal to the minimum of the function in a subinterval and the maximum of a function in an interval is always greater than or at the most equal to the maximum of the function in a subinterval. Then after substitution we will have $\omega_{1} \geqq \mu_{2}-\mu_{1}$, or we can write $\omega_{1} \geq\left|\mu_{1}-\mu_{2}\right|$. Similarly $\omega_{2} \geq\left|\mu_{4}-\mu_{3}\right|, \quad \omega_{3} \geq\left|\mu_{3}-\mu_{6}\right|, \ldots$ where $\omega_{1}, \omega_{2}, \omega_{3}, \ldots-$ represent the total variation of $f(x)$ in the intervals

$0 \leqq x \leqq \frac{\pi}{k}, \quad \frac{\pi}{k} \leq x \leq \frac{2 \pi}{k}, \frac{2 \pi}{k} \leq x \leq \frac{3 \pi}{K}, \cdots-$ respectively. 

the interval $0 \leqq x \leqq 2 \pi$ is

$$
\Omega=\omega_{1}+\omega_{2}+\omega_{3}+\cdots+\omega_{2 k} .
$$

Thus we have

(8)

$$
\begin{aligned}
& \left|b_{k}\right| \leqq \frac{1}{\pi} \frac{1}{k} \Omega: \\
& \text { Following the same method for equation (7) we get } \\
& \omega_{0} \geqq\left|\mu_{0}-\mu_{4 k-1}\right|, \quad \omega_{1} \geqq\left|\mu_{1}-\mu_{2}\right|, \quad \omega_{2} \geqq\left|\mu_{4}-\mu_{2}\right|, \ldots
\end{aligned}
$$

where $\omega_{1}, \omega_{2}, \omega_{1}, \ldots$ represent the total variation of $f(x)$ in the intervals,

$$
\frac{\pi}{2 k} \leqq x \leqq \frac{3 \pi}{2 k}, \quad \frac{3 \pi}{2 k} \leqq x \leqq \frac{5 \pi}{2 k}, \quad \frac{5 \pi}{2 k} \leqq x \leqq \frac{7 \pi}{2 k},-\cdots-\text { respectively }
$$

and $\omega$. represents the total variation in the two intervals

$$
0 \leqq x \leq \frac{\pi}{2 k} \text { and } \frac{(4 k-1) \pi}{2 k} \leqq x \leqq 2 \pi
$$

Then we have as before:

(9)

$$
\left|a_{k}\right| \leqq \frac{1}{\pi} \cdot \frac{1}{k} \cdot \Omega
$$

Thus if $\Omega$ remains finite in the interval $0 \leqq x \leqslant 2 \pi$ the quantities $\left|b_{k}\right|$ and $\left|a_{k}\right|$ will be defined as well as finite and the

$$
\lim _{k=\infty}\left|b_{k}\right|=0 \text { and } \lim _{k=\infty}\left|a_{k}\right|=0 \text {. }
$$

Hence we can conclude:

If the total rariation of e function remains finite the coefficients of 1 tg Fourier developement converge to zero.

2. Integrable in the Riemann sense. Now let us assume that the function, $f(x)$, is integrable in the Riemann sense. 

Then $\quad \int_{a}^{b} f(x) d x=\lim _{\Delta x-0} \sum_{i=1}^{n} f\left(\xi_{i}\right) \Delta x$, where $m_{i} \leqq f\left(\xi_{i}\right) \leqq M_{i}$.

We could have written equation (4) in the following form, oonsidering $\mathrm{l} / \mathrm{k}=\Delta \mathrm{x}$ :

$$
\begin{aligned}
b_{k}=\frac{I}{\pi} & \left\{\left(\mu_{1} \Delta x+\mu_{4} \Delta x+\mu_{s} \Delta x+\cdots+\mu_{4 k} \Delta x\right)\right. \\
& \left.-\left(\mu_{2} \Delta x+\mu_{3} \Delta x+\mu_{6} \Delta x+\cdots+\mu_{4 k-1} \Delta x\right)\right\} .
\end{aligned}
$$

And we have $m_{i} \leqq \mu_{i} \leqq \mathbb{M}_{i}$.

$$
\therefore \quad \lim _{k=\infty} b_{k}=\frac{1}{\pi}\left\{\frac{1}{2} \int_{0}^{2 \pi} f(x) d x-\frac{1}{2} \int_{0}^{2 \pi} f(x) d x\right\}=0 .
$$

Similarly equation (6) can be written in the form :

$$
\begin{gathered}
a_{\mathrm{k}}=\frac{1}{\pi}\left\{\left(\mu_{0} \Delta \mathrm{x}+\mu_{0} \Delta \mathrm{x}+\mu_{4} \Delta \mathrm{x}+\mu_{3} \Delta \mathrm{x}+\cdots+\mu_{4 k-3} \Delta \mathrm{x}\right)\right. \\
\left.-\left(\mu_{2} \Delta \mathrm{x}+\mu_{3} \Delta \mathrm{x}+\mu_{6} \Delta \mathrm{x}+\cdots+\mu_{4 k-1} \Delta \mathrm{x}\right)\right\} . \\
\quad \lim _{\mathrm{k}=\infty} a_{\mathrm{k}}=\frac{1}{\pi}\left\{\frac{1}{2} \int_{0}^{2 \pi} f(x) \mathrm{d} \mathrm{x}-\frac{1}{2} \int_{0}^{2 \pi} f(x) d x\right\}=0 .
\end{gathered}
$$

Thus we can conclude:

If a function is integrable in the Riemann sense the coefficients of its Fourier developement converge to zero.

3. Generalized Iipsohitz condition. Now let us assume that the function, $f(x)$, satisfies the following condition:

$$
|\Delta f|<\Delta|\Delta x|^{\alpha}
$$

where $A$ is any constant and $\alpha$ satisfies the condition $0<\alpha<1$. 

This restriotion on $f(x)$ is not nearly as great as that imposed by the so called Iipschitz condition which is that

$$
|\Delta f| \leqslant A|\Delta x|
$$

Writing $\Delta x$ as $\frac{1}{\frac{1}{k}}$ we will then have

$$
|\Delta f|<\Delta\left|\frac{1}{\mathrm{k}^{\alpha}}\right|
$$

From (5) we have, setting $\left|\mu_{1}-\mu_{2}\right| \leqq\left|\Delta f_{1}\right|, \quad\left|\mu_{4}-\mu_{3}\right| \leqq \mid \Delta f_{2}$

$$
\left|k b_{k}\right| \leqq \frac{1}{\pi}\left\{\sum_{i}^{k}\left|\Delta f_{i}\right|\right\}
$$

Then $\left|k b_{k}\right| \leqq \frac{k A}{\pi}\left|\frac{I}{k^{a}}\right|=\frac{C_{1}}{k^{\alpha-1}}$, where $C_{1}$ is a constant.

Then $\quad\left|b_{k}\right|=\frac{c_{1}}{k^{\alpha}}$ and $\quad \lim _{k=\infty}\left|b_{k}\right|=0$.

Similarly: $\left|a_{k}\right|=\frac{a_{z}}{k^{\alpha}}$ and $\lim _{k=\infty}\left|a_{k}\right|=0$.

Hence :

If 으 function satisfies a generalized Iipsohitz condition the ooefficients of its Fourler developement converge to zero. 

Chapter, II.

1. Remainder in a Fourier's series. We will now proceed to the conslderation of the accuracy of a Fourier developement taken for a finite number of terms and will develope an expression for the remainder of a Fourier's series after $n$ terms.

Let us consider the case where the function of $x$, $f(x)$, is a periodic function of period $2 \pi$, having an infinite number of derivatives of the same period.

The Fourier developement of $f(x)$ will then be of

the form;

$$
f(x)=\frac{b_{0}}{2}+b_{1} \cos x+b_{2} \cos 2 x+b_{3} \cos 3 x+-
$$

$$
+a_{1} \sin x+a_{2} \sin 2 x+a_{3} \sin 3 x+\cdots
$$

We will investigate the coefficients:

$a_{k}=\frac{1}{\pi} \int_{0}^{2 \pi} f(x)$ sin $k x d x$ and $b_{k}=\frac{1}{\pi} \int_{0}^{2 \pi} f(x) \cos k x d x$.

Integrating the first by parts we have:

$$
a_{k}=-\frac{1}{k \pi}\left[\left.f(x) \cos k x\right|_{0} ^{2 \pi}+\frac{I}{k \pi} \int_{0}^{2 \pi} f^{\prime}(x) \cos k x d x .\right.
$$

$k$ being an integer, we have $\left[\left.f(x) \cos k x\right|_{0} ^{2 \pi}=0\right.$.

Repeating the integration by parts we get:

$$
a_{k}=\frac{1}{\pi k^{2}}\left[f^{\prime}(x) \text { sin }\left.k x\right|_{0} ^{2 \pi}-\frac{1}{\pi k^{2}} \int_{0}^{2 \pi} f^{\prime \prime}(x) \text { sin } k x d x\right.
$$

Again: $\quad\left[f^{\prime}(x)\right.$ sin $\left.k x\right|_{0} ^{2 \pi}=0$. 

Repeating the integration again:

$$
a_{k}=\frac{1}{\pi k} 3\left[\left.f^{\prime \prime}(x) \cos k x\right|_{0} ^{2 \pi}-\frac{1}{\pi k^{3}} \int_{0}^{2 \pi} f^{\prime \prime \prime}(x) \cos k x d x\right.
$$

And again: $a_{k}=-\frac{1}{\pi k^{4}}\left[\left.f^{\prime \prime \prime}(x) \cos k x\right|_{0} ^{2 \pi}-\frac{1}{\pi k} 4 \int_{0}^{2 \pi} f^{n n}(x) \sin k x d x\right.$.

This process could be kept up indefinitely since

$$
\left[\left.f^{\prime \prime}(x) \cos k x\right|_{0} ^{2 \pi}, \quad\left[\left.f^{\prime \prime \prime}(x) \cos k x\right|_{0} ^{2 \pi}\right. \text {, and similar factors }\right.
$$

all drop out, being equal to zero, and since $f(x)$ has an infinite number of derivatives by hypothesis.

Carrying through the same process for $b_{k}$ we get:

$$
b_{k}=\frac{1}{\pi k}\left[\left.f(x) \sin k x\right|_{0} ^{2 \pi}-\frac{1}{\pi k} \int_{0}^{2 \pi} f^{\prime}(x) \sin k x d x\right. \text {. }
$$

But $\quad\left[f(x)\right.$ sin $\left.k x\right|_{0} ^{2 \pi}=0$.

Integrating again:

$$
b_{k}=\frac{1}{\pi k^{2}}\left[\left.f^{\prime}(x) \cos k x\right|_{0} ^{2 \pi}-\frac{1}{\pi k^{2}} \int_{0}^{2 \pi} f^{\prime \prime}(x) \cos k x d x .\right.
$$

Repeating again we have since $\left[f^{\prime}(x)\right.$ cos $\left.k x\right|_{0} ^{2 \pi}=0$,

$$
b_{k}=-\frac{1}{\pi k^{2}}\left[\left.f^{\prime \prime}(x) \sin k x\right|_{0} ^{2 \pi}+\frac{1}{\pi k} 3 \int_{0}^{2 \pi} f^{\prime \prime \prime}(x) \sin k x d x\right.
$$

And again:

$$
b_{k}=-\frac{1}{\pi k} 4\left[\left.f^{n \prime \prime}(x) \cos k x\right|_{0} ^{2 \pi}+\frac{1}{k} 4 \int_{0}^{2 \pi} f^{n \prime}(x) \cos k x d x\right. \text {, }
$$

since $\quad\left[\left.f^{\prime \prime}(x) \sin k x\right|_{0} ^{2 \pi}\right.$ and $\left[\left.f^{\prime \prime \prime}(x) \cos k x\right|_{0} ^{3 \pi}=0\right.$. 

Then the absolute value of the $n^{\text {th }}$ Fourier coefficient

will be:

$$
\left|a_{n}\right| \leqq \frac{1}{\pi n^{k}} \int_{0}^{2 \pi}\left|f^{k}(x)\right||\cos n x| d x \text {, when } k \text { is odd or }
$$

(2)

$$
\left|a_{n}\right| \leqq \frac{1}{\pi n} k \int_{0}^{2 \pi}\left|f^{k}(x)\right||\sin n x| d x \text {, when } k \text { is even. }
$$

Similarly:

$$
\left|b_{n}\right| \leqq \frac{1}{\pi n} \int_{0}^{2 \pi}\left|f^{k}(x)\right||\sin n x| d x \text {, when } k \text { is odd or }
$$

(3)

$$
\left|b_{n}\right| \leqq \frac{1}{\pi n} \int_{0}^{2 \pi}\left|e^{k}(x)\right||\cos n x| d x \text {, when } k \text { is even. }
$$

By the law of the mean we have:

$$
\left|a_{n}\right| \leqq \frac{1}{\pi n^{k}}\left|f^{k}(\bar{x})\right| \int_{0}^{2 \pi}|\cos n x| d x \text {, where } 0<\bar{x}<2 \pi \text {. }
$$

But $\quad \int_{0}^{2 \pi}|008 n x| d x=\frac{1}{n} \cdot 4 n-4$.

(4) $\therefore\left|a_{n}\right|=\frac{4}{\pi n^{k}}\left|f^{k}(\bar{x})\right|$. And similarly $\left|b_{n}\right|=\frac{4}{\pi n^{k}}\left|f^{k}(\bar{x})\right|$.

Then in order to have a good representation of $f(x)$ by its Fourier developement the remainder after $n$ terms of the series must approach zero. From (1), since $|\sin k x| \leqq I$ and $|\cos k x| \leqslant 1$, we can write the remainder:

$$
R_{n} \leqq\left|a_{n}\right|+\left|b_{n}\right|+\left|a_{n-1}\right|+\left|b_{n-1}\right|+\cdots
$$

Substituting the values determined for $\left|a_{n}\right|,\left|b_{n}\right|$, etc.,

(5) $R_{n} \leqq\left|f^{k}(\dot{x})\right| \frac{4}{\pi}\left\{\frac{1}{n^{k}}+\frac{1}{n^{k}}+\frac{1}{(n+1)} k+\frac{1}{(n+1)^{k}}+\cdots-\right\}$.

$$
R_{n} \leqq\left|f^{k}(\bar{x})\right| \frac{8}{\pi}\left\{\frac{1}{n}+\frac{1}{(n+1)^{k}}+\frac{1}{(n+2)^{k}}+\cdots-\cdot\right\} \text {. }
$$



I.et

$$
F(n, k)=\frac{1}{n^{k}}+\frac{1}{(n+1)^{k}}+\frac{1}{(n+2)^{k}}+\cdots
$$

Then

$$
R_{n}(x)=\theta\left[\frac{8}{\pi}\left|f^{k}(\bar{x})\right| F(n, k)\right] \text {, where } 0<\theta<1 \text {, or }
$$

$$
R_{n}(x) \leqq\left[\frac{8}{\pi} M_{k} F(n, k)\right],
$$

where $\mathbb{M}_{k}$ is the maximum value of $\left|f^{k}(\bar{x})\right|$.

We can then judge the oharacter of our remainder by computing $\left|f^{k}(\bar{x})\right|$ for a number of values of $k$ and noting what choice of $k$ will make $R_{n}(x)$ the smallest. For this purpose we will tabulate $F(n, k)$ for a number of values of $n$ and $k$.

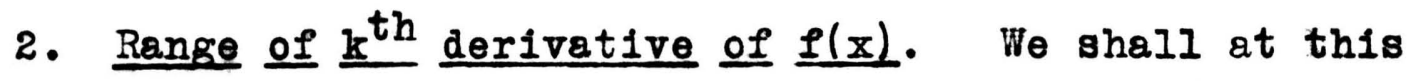
point prove a rather striking theorem:

If a function of $x, f(x)$, is a periodic function possessing an infinite number of derivatives having the same period as $f(x)$, and if $f(x)$ cannot be developed in a finite Fourier's series, then after a certain point the maximum value of the $\mathrm{k}^{\text {th }}$ derivative of $f(x)$ will increase faster than an exponential function of the form $n^{k}$.

$$
\text { Let } M_{k}=\text { maximum value of }\left|f^{k}(x)\right| \text {. }
$$

Then from equations (4):

$$
\left|a_{n}\right| \leqq \frac{4}{\pi n^{k}} M_{k} \quad \text { and } \quad\left|b_{n}\right| \leqq \frac{4}{\pi n} k M_{k}
$$

Rewriting :

$$
\left|a_{n}\right| \leqq \frac{4}{\pi} \frac{M_{k}}{n^{k}} \quad \text { and } \quad\left|b_{n}\right| \leqq \frac{4}{\pi} \frac{M_{k}}{n^{k}}
$$

We see at once that if $\left|a_{n}\right|=0$ or $\left|b_{n}\right|=0$ we would know nothing definite about the conduct of $\mathbb{M}_{k}$. It might increase as fast as $\mathrm{n}^{\mathrm{k}}$ or it might not, just as the case happened 

to be.

But our hypothesis was that $\left|a_{n}\right|>0$ or $\left|b_{n}\right|>0$, or both, for an infinite number of values of $n$. This will then force $M_{k}$ to increase faster than $n^{k}$ increases for any value of $n$ or otherwise the fraction $\mathrm{M}_{\mathrm{k}} / \mathrm{n}^{\mathrm{k}}$ will approach zero and hence violate the hypothesis of our theorem, since if $\mathrm{M}_{\mathrm{K}} / \mathrm{n}^{\mathrm{k}}$ approaches zero $\left|a_{n}\right|$ and $\left|b_{n}\right|$ must also approach zero, each being less than $M_{k} / n^{k}$.

Thus if we have a function that satisfies the conditions of our theorem and we assign integral values to $k, 1,2,3,-$ then after a certain value of $\mathrm{k}$ is reached, $\mathrm{M}_{\mathrm{k}}$ w1ll increase faster than an exponential function of the type $\mathrm{n}^{\mathrm{k}}$. As a corollary to the above theorem we can state the following:

If $\mathbb{M}_{k}$ is bounded by a function of the type $\mathrm{m}^{\mathrm{k}}, f(x)$ is a trigonometric polynomial of order at most equal to $\mathrm{m}$.

For the reasoning shows that then $a_{n}=0$ and $b_{n}=0$ for $n>m$ and the function would thus be a trigonometric polynomial of order at most equal $m$.

A simple example of such a function is the function $f(x)=\sin 2 x$. Then $f^{\prime}(x)=2 \cos 2 x, f^{n}(x)=-4 \sin 2 x$, $f^{\prime \prime}(x)=-2^{3}$ oos $2 x, f^{\prime \prime \prime}(x)=2^{4}$ sin $2 x$, etc.,

and thus we have $\left|f^{k}(x)\right| \leqq 2^{k}$. Therefore $\mathbb{M}_{k}=2^{k}$, which verifies our corollary.

3. Table of palpes of $F(n, k)$. We will now tabulate the function $F(n, k)$ for values of $n$ ranging from $I$ to 20 and for $k$ ranging from 1 to 20. In computing this table I have made use of some results given by Stieltjes ${ }^{12}$. 

n $F(n, 2) \quad F(n, 3) \quad F(n, 4) \quad F(n, 5) \quad F(n, 6)$

$\begin{array}{llllll}1 & 1.6449341 & 1.2020569 & 1.0823232 & 1.0369278 & 1.017343062\end{array}$

$\begin{array}{llllll}2 & .6449341 & .2020569 & .0823232 & .0369278 & .017343062\end{array}$

$\begin{array}{llllll}3 & .3949341 & .0770569 & .0198232 & .0056778 & .001718062\end{array}$

$\begin{array}{llllll}4 & .2838229 & .0400199 & .0074775 & .0015625 & .000346320\end{array}$

$\begin{array}{llllll}5 & .2213229 & .0243949 & .0035713 & .0005860 & .000102180\end{array}$

$\begin{array}{llllll}6 & .1813229 & .0163949 & .0019713 & .0002660 & .000038180\end{array}$

$\begin{array}{llllll}7 & .1535452 & .0117652 & .0011997 & .0001374 & .000016747\end{array}$

$\begin{array}{llllll}8 & .1331371 & .0088498 & .0007832 & .0000779 & .000008248\end{array}$

$\begin{array}{llllll}9 & .1175121 & .0068967 & .0005391 & .0000473 & .000004434\end{array}$

$\begin{array}{llllll}10 & .1051664 & .0055249 & .0003866 & .0000304 & .000002553\end{array}$

$\begin{array}{llllll}11 & .0951664 & .0045249 & .0002866 & .0000204 & .000001553\end{array}$

$\begin{array}{llllll}12 & .0869019 & .0037736 & .0002183 & .0000142 & .000000989\end{array}$

$\begin{array}{llllll}13 & .0799575 & .0031949 & .0001701 & .0000121 & .000000655\end{array}$

$\begin{array}{llllll}14 & .0740403 & .0027397 & .0001351 & .0000075 & .000000448\end{array}$

$\begin{array}{llllll}15 & .0689383 & .0023753 & .0001091 & .0000056 & .000000316\end{array}$

$\begin{array}{llllll}16 & .0644938 & .0020790 & .0000893 & .0000043 & .000000229\end{array}$

$\begin{array}{llllll}17 & .0605876 & .0018349 & .0000741 & .0000034 & .000000170\end{array}$

$\begin{array}{llllll}18 & .0571274 & .0016313 & .0000621 & .0000027 & .000000129\end{array}$

$\begin{array}{llllll}19 & .0540409 & .0014600 & .0000526 & .0000021 & .000000090\end{array}$

$\begin{array}{llllll}20 & .0512709 & .0013141 & .0000449 & .0000017 & .000000069\end{array}$ 


n $\quad F(n, \eta)$
$F(n, 8)$
$F(n, 9)$
$F(n, 10)$

$\begin{array}{lllll}1 & 1.008349277 & 1.00407735620 & 1.002008392826 & 1.000994575128\end{array}$

$2 \quad .008349277 \quad .00407735620$

$.002008392826 \quad .000994575128$

$3 \quad .000536777 \quad .00017110620$

$.000055267826 \quad .000018012628$

$\begin{array}{lll}4 & .000079530 \quad .00001869047\end{array}$

$.000004462563 \quad .000001077541$

$5 \quad .000018495$

.00000343228

.000000648033

.000000123909

$6 \quad .000005695$

.00000087228

.000000136033

.000000021509

$7 \quad .000002123$

.00000027691

.000000036805

.000000004971

$\begin{array}{lll}8 & .000000909 & .00000010363\end{array}$

.000000012051

.000000001435

$9 \quad .000000433$

.00000004401

.000000004599

.000000000504

$10 \quad .000000224$

.00000002078

.000000002018

.000000000218

11.00000012

.00000001078

.000000001018

.000000000118

$12 \quad .00000007$

.00000000612

.000000000955

.000000000080

$18 \quad .000000046$

.00000000380

.000000000402

.000000000064

$14 \quad .000000031$

.00000000258

.000000000308

.000000000057

$16 \quad .000000022$

.00000000192

.000000000260

.000000000054

$16.000000017 \quad .00000000152$

.000000000234

.000000000053

$17 \quad .00000001$

.00000000129

.000000000220

.000000000053

18.00000001

.00000000115

.000000000212

$19 \quad .000000010$

$20 \quad .000000009$ 


n $\quad F(n, 11)$
$F(n, 12)$
$F(n, 13)$
$F(n, 14)$

$1 \quad 1.000494188604 \quad 1.0002460865533$

$1.0001227133475 \quad 1.0000612481350$

$\begin{array}{lllll}2 & .000494188604 & .0002460865533 & .0001227133475 & .0000612481350\end{array}$

$\begin{array}{llllll}3 & .000005907354 & .0000019459283 & .0000006430350 & .0000002129788\end{array}$

$\begin{array}{llllll}4 & .000000262325 & .0000000642519 & .0000000158096 & .0000000039037\end{array}$

$\begin{array}{lllllll}5 & .000000023917 & .0000000046499 & .0000000009091 & .0000000001786\end{array}$

$\begin{array}{llllll}6 & .000000003437 & .0000000005539 & .0000000000899 & .0000000000148\end{array}$

$\begin{array}{lllllll}7 & .000000000681 & .0000000000945 & .0000000000134 & .0000000000021\end{array}$

$\begin{array}{llllll}8 & .000000000175 & .0000000000224 & .0000000000031 & .0000000000007\end{array}$

$\begin{array}{lllllll}9 & .000000000059 & .0000000000081 & .0000000000013 & .0000000000005\end{array}$

$\begin{array}{llll}10 & .000000000027 & .0000000000046 \quad .0000000000010\end{array}$

$\begin{array}{llll}11 & .000000000017 & .0000000000036 & .0000000000009\end{array}$

$\begin{array}{lll}12 & .000000000014 \quad .0000000000033\end{array}$

$\begin{array}{lll}13 & .000000000012 \quad .0000000000032\end{array}$

$14 \quad .000000000012$

$\begin{array}{ll}15 & .000000000012\end{array}$

$16 \quad .000000000011$ 



$\begin{array}{cccc}\mathrm{n} & F(n, 15) & F(n, 16) & F(n, 17) \\ 1 & 1.0000305882363 & 1.000015282259408 & 1.000007637197637 \\ 2 & .0000305882363 & .000015282259408 & .000007637197637 \\ 3 & .0000000706582 & .000000023470346 & .000000007803106 \\ 4 & .0000000009665 & .000000000239773 & .000000000059582 \\ 5 & .0000000000353 & .000000000006942 & .000000000001375 \\ 6 & .0000000000026 & .000000000000379 & .000000000000064 \\ 7 & .0000000000005 & & \\ 8 & .0000000000003 & & \end{array}$

n $\quad F(n, 18) \quad F(n, 19) \quad F(n, 20)$

$11.00000381729326499 \quad 1.00000190821271655 \quad 1.000000953962033872$

$\begin{array}{llll}2 & .00000381729326499 & .00000190821271655 & .000000953962033872\end{array}$

$\begin{array}{llll}3 & .00000000259599937 & .00000000086408374 & .000000000287717466\end{array}$

$\begin{array}{lllll}4 & .00000000001482458 & .00000000000369214 & .000000000000920267\end{array}$

$\begin{array}{lllll}5 & .00000000000027267 & .00000000000005416 & .0000000000000107728\end{array}$

$\begin{array}{llll}6 & .00000000000001052 & .00000000000000173 & .0000000000000002871\end{array}$ 

4. Comparison of results. As has already been mentioned in the introduction Jackson has found that in the case of a periodic function of period $2 \pi$ whose $(k-1)$ th derivative satisfies a Iipsohita condition the remainder after $n$ terms of its Fourier's series is:

$$
R_{n}(x) \leqq 36 \lambda \frac{\log n}{n^{K}}
$$

If $\mathrm{k}$ is odd the factor 36 can be replaced by 12 .

We will compare this with the result which ve have just derived, namely,

$$
R_{n}(x) \leqq \frac{8}{\pi} \quad M_{k} F(n, k),
$$

$\mathbf{M}_{\mathrm{k}}$ being the maximum value assumed by the $\mathrm{k}^{\text {th }}$ derivative of $f(x)$ in the interval 0 to $2 \pi$.

We see at once that $8 / \pi<12$ and $\mathbb{k}_{k} \leqq \lambda, \lambda$ being the constant of the Iipschitz condition. If we let $\frac{\log _{n} n}{n^{k}}=J(n, k)$, we have left to compare only $J(n, k)$ and $F(n, k)$. When $F(n, k)<J(n, k)$ our result gives a more accurate expression for $R_{n}$ and when $F(n, k)>J(n, k)$ Jackson's result is the better. Since we have already constructed a table of values for $F(n, k)$ we have but to make a corresponding table for $J(n, k)$ and compare the two. In order to compare the functions accurately for large values of $n$ and $k$ it would be necessary to compute $F(n, k)$ to a much greater number of decimal places than has been done in our table. However, in any particular problem in which the development of a function arises, the development, to be of any value, must be such that it will be necessary to use only a few terms of $1 t$. Thus it will not be necessary to consider large values of $n$. However it will be advantage ous to 

let $\mathrm{k}$ be as large as possible. In making the following comparison I have considered values of $\mathrm{n}$ from 3 to 8 and of $\mathrm{k}$ from 2 to 20 inclusive, this being the extent of $k$ in our table of $F(n, k)$.

n

$3 F(n, k)>J(n, k)$ for $k=2$ to $k=8, F<J$ for $k=9$ to $k=26$

$4 F(n, k)>J(n, k)$ for $k=2$ to $k=6$, $F<J$ for $k=7$ to $k=2 C$

$5 F(n, k)>J(n, k)$ for $k=2$ to $k=5, F<J$ for $k=6$ to $k=2 C$

$6 F(n, k)>J(n, k)$ for $k=2$ to $k=5$, $F<J$ for $k=6$ to $k=2 C$

$7 \quad F(n, k)>J(n, k)$ for $k=2$ to $k=6, F<J$ for $k=6$ to $k=2 C$

$8 \mathrm{~F}(\mathrm{n}, \mathrm{k})>\mathrm{J}(\mathrm{n}, \mathrm{k})$ for $\mathrm{k}=2$ to $\mathrm{k}=6, \mathrm{~F}<\mathrm{J}$ for $\mathrm{k}=6$ to $\mathrm{k}=2 \mathrm{C}$

As far as I can judge, as $k$ is increased further

$\mathrm{F}(\mathrm{n}, \mathrm{k})$ still remains less than $\mathrm{J}(\mathrm{n}, \mathrm{k})$. Since in general $\mathrm{k}$ will be chosen quite large we see by the above table that our result has some advantage over that of Jackson's for this rance of $n$. 



\section{Chapter III.}

1. Remainder in a Iegendre's series. We will now consider the developement of a function of $x, f(x)$, in a series of Zonal Harmonics or what are usually called legendre's polynomials, in the interval from $x=-1$ to $x=+1$.

We have the developement:

$$
f(x)=A_{0} P(x)+A_{1} P_{1}(x)+A_{2} P_{2}(x)+\cdots+A_{m} P_{m}(x)+-
$$

where

$$
A_{m}=\frac{2 m+1}{2} \int_{-1}^{+1} f(x) P_{m}(x) d x, \quad m=1,2,3, \ldots
$$

If the series (1) converges it will be sufficient to study the convergence of the series of coefficients in investigating this development since we know that $\left|P_{m}(x)\right| \leqq 1$.

In doing this we will use a method somewhat similar to the one used in the case of a Fourier development.

Integrating (2) by parts we will have:

$$
A_{m}=\frac{2 m+1}{2}\left\{\left[\left.\int_{-1}^{x} P_{m}(x) d x f(x)\right|_{-1} ^{+1}-\int_{-1}^{+1}\left(\int_{-1}^{x} P_{m}(x) d x\right) f^{\prime}(x) d x\right\}\right.
$$

From Iegendre's equation,

$$
\frac{d}{d x}\left(I-x^{2}\right) \frac{d P_{m}}{d x}(x)+m(m+I) P_{m}(x)=0 \text {, }
$$

we have $\int_{-1}^{x} P_{m}(x) d x=-\frac{1}{m(m+1)}\left(1-x^{2}\right) \frac{d P_{m}}{d x}(x), \quad m \neq 0$.

Hence (3) becomes, the first term being equel to zero, 

(4) $A_{m}=\frac{2 m+1}{2 m(m+1)} \int_{-1}^{+1} \frac{d P_{m}(x)}{d x}\left[\left(1-x^{2}\right) f^{\prime}(x)\right] d x$.

The work can be very much simplified by making the following substitutions: Iet

$$
\begin{aligned}
& F_{0}(x)=f(x), \quad F_{1}(x)=\left(1-x^{2}\right) \frac{d}{d x} f(x), \\
& F_{2}(x)=\frac{d}{d x} F_{1}(x), \quad F_{3}(x)=\left(1-x^{2}\right) \frac{d}{d x} F_{2}(x), \\
& F_{4}(x)=\frac{d}{d x} F_{3}(x), \\
& \text { etc. }
\end{aligned}
$$

Equation (4) then becomes:

$$
A_{m}=\frac{2 m+1}{2 m(m+1)} \int_{-1}^{+1} \frac{d}{d x} P_{m}(x) F_{I}(x) d x
$$

Integrating by parts again:

$$
A_{m}=\frac{2 m+1}{2 m(m+I)}\left\{\left[\left.P_{m}(x) F_{I}(x)\right|_{-1} ^{+1}-\int_{-1}^{+1} P_{m}(x) \frac{d}{d x} F_{I}(x) d x\right\} \cdot\right.
$$

Again the first term drops out and we have:

$$
A_{m}=-\frac{2 m+1}{2 m(m+1)} \int_{-1}^{+1} P_{m}(x) F_{2}(x) d x .
$$

Prooeeding as before we get:

$$
\begin{aligned}
A_{m} & =-\frac{2 m+1}{2 m(m+I)}\left\{\left[\left.\int_{-1}^{x} P_{m}(x) d x F_{2}(x)\right|_{-1} ^{+1}-\int_{-1}^{+1}\left(\int_{-1}^{x} P_{m}(x) d x\right) \frac{d}{d x} F_{2}(x) d x\right.\right. \\
& =\frac{2 m}{\left.2 m^{2}+\frac{1}{m+1}\right)^{2}} \int_{-1}^{+1} \frac{d}{d x} P_{m}(x)\left[\left(1-x^{2}\right) \frac{d}{d x} F_{2}(x)\right] d x .
\end{aligned}
$$





$$
A_{m}=\frac{2 m+1}{2 m^{2}(m+1)} 2 \int_{-1}^{+1} \frac{d}{d x} P_{m}(x) F_{3}(x) d x
$$

In the same way we get:

$$
A_{m}=-\frac{2 m+1}{2 m^{2}(m+1)^{2}} \int_{-1}^{+1} P_{m}(x) F_{4}(x) d x
$$

Hence after integration by parts $2 k-1$ times we will have:

$$
A_{m}=\frac{2 m+1}{2 m^{k}(m+1)} k \int_{-1}^{+1} \frac{d}{d x} P_{m}(x) F_{2 k-1}(x) d x, \quad k=1,2,3, \ldots
$$

and after integration by parts $2 \mathrm{k}$ times,

$$
A_{m}=-\frac{2 m+1}{2 m^{k}(m+1)} k \int_{-1}^{+1} P_{m}(x) F_{2 k}(x) d x, \quad k=0,1,2, \ldots
$$

We have however that

$$
\begin{aligned}
& F_{1}(x)=\left(1-x^{2}\right) f^{\prime}(x), F_{2}(x)=\left(1-x^{2}\right) f^{\prime \prime}(x)-2 x f^{\prime}(x), \\
& F_{3}(x)=\left(1-x^{2}\right)^{2} f^{\prime \prime \prime}(x)-4 x\left(1-x^{2}\right) f^{\prime \prime}(x)-2\left(1-x^{2}\right) f^{\prime}(x) .
\end{aligned}
$$

And similarly:

$$
F_{k}(x)=c_{1}(x) f^{k}(x)+c_{2}(x) f^{k-f(x)}+c_{3}(x) f^{k-f}(x)+-+c_{k} f^{\prime}(x),
$$

where $c_{i}(x)$ are integral rationel functions of $x$.

$$
\text { Now suppose that the } k^{\text {th }} \text { derivative of } f(x) \text { is }
$$

bounded, i.e.,

$$
\left|f^{k}(x)\right|<N
$$

where $\mathbb{N}$ is a positive quantity. Then $F_{k}(x)$ will also be 

bounded. Throughout the discussion it must be remembered that we are considering only the values of $x$ in the interval -1 to +1 . Call the maximum value of $\left|F_{k}(x)\right|$ in the interval, $M_{k}$. Then if $k$ is an even number equal, say, to $2 n$ we will have :

$$
\left|A_{m}\right| \leqq \frac{2 m+1}{2 m^{n}(m+1)} n M_{k} \int_{-1}^{+1}\left|P_{m}(x)\right| d x
$$

or if $k$ is an odd number, $k=2 n-1$,

$$
\left|A_{m}\right| \leqq \frac{2 m+1}{2 m^{n}(m+1)} n M_{k} \int_{-1}^{+1}\left|\frac{d}{d x} P_{m}(x)\right| d x
$$

Thus we have an upper bound for $\left|A_{m}\right|$. We need only consider one of these namely the first. To determine the value of the integral $\int_{-1}^{+1}\left|P_{m}(x)\right| d x$ we will use Schwarz's inequality which states that

$$
\left(\int_{a}^{b} f(x) \cdot \varphi(x) d x\right)^{2} \leqq \int_{a}^{b}(f(x))^{2} d x \cdot \int_{a}^{b}(\varphi(x))^{2} d x,
$$

where $f(x)$ and $\varphi(x)$ are two real and continuous functions. Now if we set $f(x)=I$ and $\varphi(x)=\left|P_{m}(x)\right|$ we have:

$$
\left(\int_{-1}^{+1}\left|P_{m}(x)\right| d x\right)^{2} \leqq 2 \int_{-1}^{+1}\left(P_{m}(x)\right)^{2} d x \text { since }\left(\left|P_{m}(x)\right|\right)^{2}=\left(P_{m}(x)\right)^{2}
$$

But $\int_{-1}^{+1}\left(P_{m}(x)\right)^{2} d x=\frac{2}{2 m+1}$. Hence $\int_{-1}^{+1}\left|P_{m}(x)\right| d x<\frac{2}{\sqrt{2 m+1}}$. 

Since $\left|P_{m}(x)\right|>\left(P_{m}(x)\right)^{2}$ throughout the interval

- I to +1 , we can say that

$$
\frac{2}{2 m+1}<\int_{-1}^{+1}\left|P_{m}(x)\right| d x \leqq \frac{2}{\sqrt{2 m+1}} \text {. }
$$

We have that the remainder after $m$ terms of our

series is

$$
R_{m} \leqq\left|A_{m}\right|+\left|A_{m+1}\right|+\left|A_{m+2}\right|+\cdots+\left|A_{m+p}\right|+\cdots
$$

This will then become:

$$
R_{m} \leqq M_{k}\left\{\frac{(2 m+1)^{\frac{1}{2}}}{m^{n}(m+1)^{n}}+\frac{(2 m+3)^{\frac{1}{2}}}{(m+1)^{n}(m+2)^{n}}+\frac{(2 m+5)^{\frac{1}{2}}}{(m+2)^{n}(m+3)^{n}} n+-\right\} \text {. }
$$

where $2 n$ is again equal to $k$.

The series

$$
\frac{(2 m+1)^{\frac{1}{2}}}{m^{n}(m+1)^{n}}+\frac{(2 m+3)^{\frac{1}{2}}}{(m+1)^{n}(m+2)^{n}}+\frac{(2 m+5)^{\frac{1}{2}}}{(m+2)^{1}(m+3)^{n}}+\cdots
$$

is a convergent series for values of $n \geqq l$ and $m \geqq l$. It converges very rapialy as $n$ is taken larger and larger. Since it will be In general possible to assign a moderately large value to $n$ it will only be necessary to compute a few terms of the series to obtain a result accurate enough for all purposes. $\mathbb{M}_{k}$ then being known or computed we will have an upper bound for the remainder after n terms of a Iegendre's series. 



\section{Chapter IV.}

1. Remainder in series of Bessel's functions. Iet to now consider the development of a function in a series of Bessel's funotions of the zeroth order, between the limits 0 and $I$.

We have:

$$
f(x)=A_{1} J_{0}\left(\mu_{1} x\right)+A_{2} J_{0}\left(\mu_{2} x\right)+A_{3} J_{0}\left(\mu_{3} x\right)+\cdots
$$

where $\mu_{1}, \mu_{2}, \mu_{3},-$ - - are the successive roots of the transcendental equation $J_{0}(\mu x)=0$ and

$$
A_{k}=\frac{2}{J_{I}^{2}\left(\mu_{k}\right)} \int_{0}^{1} x f(x) J_{0}\left(\mu_{k} x\right) d x \text {. }
$$

We will use the same method that we hbre used in discussing the other series.

Integrating $A_{\mathrm{k}}$ by parts we have:

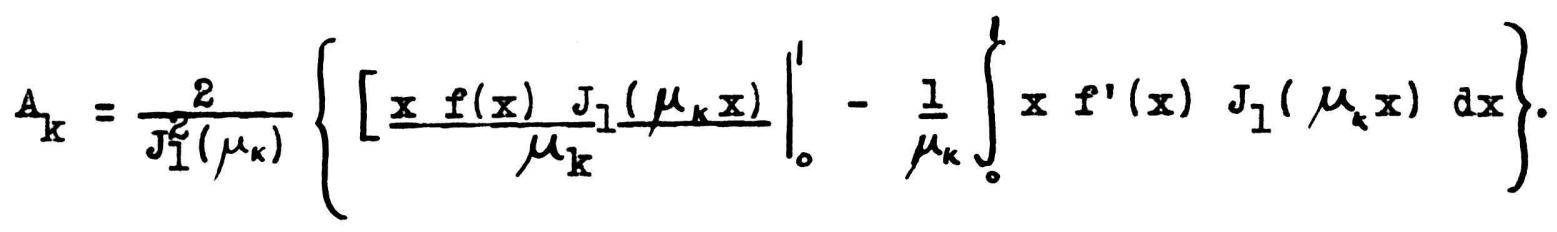

We will at this point simplify our work a great deal by sotting up the following auxiliary functions:

$$
\begin{aligned}
& F_{0}(x)=f(x), \quad F_{1}(x)=\frac{f^{\prime}(x)}{x}, \quad F_{2}(x)=\frac{F f(x)}{x} . \\
& -F_{n-1}(x)=\frac{F_{n-2}^{\prime}(x)}{x}, F_{n}(x)=\frac{F_{n-1}^{\prime}(x)}{x} .
\end{aligned}
$$

It will be necessary to impose the following conditions upon these functions:

$$
F_{0}(I)=F_{I}(I)=F_{2}(I)=-\cdots=F_{n-I}(I)=0 .
$$

With these conditions we will then have: 

$-31-$

$$
\begin{aligned}
& \quad\left[\left.\frac{x f(x) J_{l}\left(\mu_{k} x\right)}{\mu_{k}}\right|_{0} ^{1}=0\right. \text { and consequently } \\
& A_{k}=\frac{-2}{J_{1}^{2}\left(\mu_{k}\right)} \frac{1}{\mu_{k}} \int_{0}^{1} x^{2} F_{1}(x) J_{1}\left(\mu_{k} x\right) d x .
\end{aligned}
$$

Integrating by parts again:

$$
\begin{aligned}
A_{k} & =\frac{-2}{J_{1}^{2}\left(\mu_{k}\right)} \frac{1}{\mu_{k}}\left\{\left[\left.\left(\int_{0}^{x} x^{2} J_{1}\left(\mu_{k} x\right) d x\right) F_{1}(x)\right|_{0} ^{1}-\int_{0}^{1}\left[\int_{0}^{x} x^{2} J_{1}\left(\mu_{k} x\right) d x\right] F_{I}^{\prime}(x) d x\right\}\right. \\
& =\frac{-2}{J_{1}^{2}\left(\mu_{k}\right)} \frac{I}{\mu_{k}}\left\{\frac{\left.\left.x^{2} J_{2}\left(\mu_{k} x\right) F_{I}(x)\right|_{0} ^{1}-\frac{1}{\mu_{k}} \int_{0}^{1} x^{2} J_{2}\left(\mu_{k} x\right) F_{1}^{\prime}(x) d x\right\} .}{}\right. \\
& =\frac{2}{J_{1}^{2}\left(\mu_{k}\right)} \frac{1}{\mu_{k}^{2}} \int_{0}^{1} x^{2} J_{2}\left(\mu_{k} x\right) F_{1}^{\prime}(x) d x \\
& =\frac{2}{J_{1}^{2}\left(\mu_{k}\right)} \frac{I}{\mu_{k}^{2}} \int_{0}^{1} F_{2}(x) x^{3} J_{2}\left(\mu_{k} x\right) d x .
\end{aligned}
$$

Repeating the integration by parts:

$$
\begin{aligned}
A_{\mathrm{k}} & =\frac{2}{J_{1}^{2}\left(\mu_{k}\right)} \frac{1}{\mu_{k}^{2}}\left\{\left[\left.F_{2}(x) \int_{0}^{x} x^{3} J_{2}\left(\mu_{k} x\right) d x\right|_{0} ^{1}-\int_{0}^{1}\left(\int_{0}^{x} x^{3} J_{2}\left(\mu_{k} x\right) d x F_{2}^{\prime}(x) d x\right\}\right.\right. \\
& =\frac{2}{J_{1}^{2} \frac{1}{\left.\mu_{k}\right)}} \frac{1}{\mu_{k}^{2}}\left\{\left.\frac{F_{2}(x) x^{3} J_{3}\left(\mu_{k} x\right)}{\mu_{k}}\right|_{0} ^{1}-\frac{1}{\mu_{k}} \int_{0}^{1} F_{2}^{\prime}(x) x^{3} J_{3}\left(\mu_{k} x\right) d x\right\} . \\
& =\frac{-2}{J_{1}^{2}\left(\mu_{k}\right)} \frac{1}{\mu_{k}^{3}} \int_{0}^{1} F_{3}(x) x^{4} J_{3}\left(\mu_{k} x\right) d x .
\end{aligned}
$$



Repeating this process $m$ times we will finally get:

$$
A_{k}=\frac{(-1)^{m} 2}{J_{1}^{2}\left(\mu_{k}\right)} \frac{1}{\mu_{k}^{m}} \int_{0}^{1} F_{m}(x) x^{m+1} J_{m}\left(\mu_{k} x\right) d x
$$

By the law of the mean we can say:

$$
A_{k}=\frac{(-I)^{m} 2}{J_{1}^{2}\left(\mu_{k}\right)} \frac{I}{\mu_{k}^{m}} F_{m}(\bar{x}) J_{m}\left(\mu_{k} \bar{x}\right) \int_{0}^{1} x^{m+1} d x \text {, where } 0 \leqq x \leqq 1 .
$$

Then $\quad A_{k}=\frac{2}{J_{I}^{2}\left(\mu_{k}\right)} \frac{I}{\mu_{k}^{m}} \frac{\operatorname{MF}_{m}(x) \cdot M_{m}\left(\mu_{k} x\right)}{m+2}$

where by $\mathrm{MF}_{m}(x)$ and $\mathrm{MJ}_{m}\left(\mu_{k} x\right)$ we mean the maximum values assumed by $F_{m}(x)$ and $J_{m}\left(\mu_{x} x\right)$ respectively in the interval $x=0$ to $x=1$. We have however that $\left|J_{m}(x)\right| \leqq I$ for all values of $x$. For Whittaker's Modern Analysis, article 153, gives us an expression for the Bessel functions in the form of an integral. The following formula is developed:

$$
J_{m}(x)=\frac{1}{\pi} \int_{0}^{\pi} \cos (m \theta-x \sin \theta) d \theta .
$$

But since the value of the integrand can never exceod unity we can conclude that $\left|J_{m}(x)\right| \leqq I$ for all values of $x$. It can also be shown that the first maximum of $J_{m}(x)$ is the largest one. Then in considering the convergence of the series

$$
f(x)=A_{1} J_{0}\left(\mu_{1} x\right)+A_{2} J_{0}\left(\mu_{2} x\right)+A_{3} J_{0}\left(\mu_{2} x\right)+\cdots
$$

we can consider instead the series

$$
f(x) \leqslant\left|A_{1}\right|+\left|A_{2}\right|+\left|A_{3}\right|+\cdots+\left|A_{n}\right|+\cdots
$$



Hence we have:

$$
\begin{aligned}
& R_{n}(x) \leqq\left|A_{n}\right|+\left|A_{n-1}\right|+\left|A_{n-2}\right|+\cdots- \\
& R_{n}(x) \leqq \frac{2}{m+2}{ }_{m}^{M F}(x)\left\{\frac{M J_{m}\left(\mu_{n} x\right)}{\mu_{n}^{m} J_{I}^{2}\left(\mu_{n}\right)}+\frac{M_{m}\left(\mu_{n+1} x\right)}{\mu_{n+1}^{m} J_{I}^{2}(\mu)}+\frac{M_{m}\left(\mu_{n+2} x\right)}{\mu_{n+2}^{m} J_{I}^{2}\left(\mu_{n+2}\right)}+--\right\}
\end{aligned}
$$

Or we could write:

$$
\mathrm{R}_{n}(x) \leqq \frac{2}{m+2} \mathrm{MF}_{m}(x) \cdot \mathrm{MJ}_{m}\left(\mu_{n} x\right)\left\{\frac{1}{\mu_{n}^{m} J_{I}^{2}\left(\mu_{n}\right)}+\frac{1}{\mu_{n+1}^{m} J_{I}^{2}\left(\mu_{n+1}\right)}+--\right\} .
$$

Since $\mu_{n}, \mu_{n+1}, \mu_{n+2}$, - - are successive roots of

the equation $J_{0}(x)=0, J_{1}^{2}\left(\mu_{n}\right), J_{1}^{2}\left(\mu_{n+1}\right), J_{1}^{2}\left(\mu_{n+2}\right),-$ are all greater than zero.

If then the series

$$
\frac{1}{\mu_{n}^{m} J_{I}^{2}\left(\mu_{n}\right)}+\frac{I}{\mu_{n+1}^{m} J_{I}^{2}\left(\mu_{n+1}\right)}+\frac{I}{\mu_{n+2}^{m} J_{I}^{2}\left(\mu_{n+2}\right)}+-\cdot-
$$

is a convergent series it will be possible to compute an upper bound for the values of $R_{n}(x)$.

We will now proceed to show that the above series is convergent for values of $m>2$, and to obtain an upper limit for its value.

We have the following asymptotic formulal5:

(1) $J_{0}(x)=\frac{2 \cos (\pi / 4-x)}{\sqrt{2 \pi x}}+\theta 2 \sqrt{\frac{2}{\pi}} g^{2} e^{-1} x^{-5 / 2}$ where $-1<\theta<1$ and $g<3.5$. 

Then $\quad x J_{0}(x)=\frac{2 x^{\frac{1}{2}} \cos (\pi / 4-x)}{\sqrt{2 \pi}}+\theta 2 \sqrt{\frac{2}{\pi}} g^{2} e^{-1} x^{-\frac{3}{2}}$

and $x J_{0}(x)=\frac{d}{d x}\left(x J_{l}(x)\right)$ by a well known formula.

Integrating between two indefinite limits, $a$ and $\lambda$ we will have:

And then

$$
\int_{a}^{\lambda} x J_{0}(x) d x=\lambda J_{I}(\lambda)-a J_{I}(a)
$$

$$
\lambda J_{I}(\lambda)-a J_{I}(a)=\frac{2}{\sqrt{2 \pi}} \int_{a}^{\lambda} x^{\frac{1}{2}} \cos (\pi / 4-x) d x-\left[\left.\theta 4 \sqrt{\frac{2}{\pi}} g^{2} e^{-1} x^{-\frac{1}{2}}\right|_{a} ^{\lambda}\right.
$$

Iet us call the second member of the right hand side of this equation $R$. $R$ then tends toward zero as $\lambda$ and a increase. If now we could choose a as a root of $J_{l}(x)=0$ we would have an expression for $\lambda J_{1}(\lambda)$. However if we choose a not equal to such a root it amounts only to adding a constant to $\lambda J_{1}(\lambda)$.

Integrating the first member of the right hand side of equation (2) by parts we get, leaving off for the moment the constant term:

$$
\begin{aligned}
& \int x^{\frac{1}{2}} \cos (\pi / 4-x) d x=-\sin \left(\frac{\pi}{4}-x\right) x^{\frac{1}{2}}+\frac{1}{2} \int x^{-\frac{1}{2}} \sin \left(\frac{\pi}{4}-x\right) d x \\
& =-\sin \left(\frac{\pi}{4}-x\right) x^{\frac{1}{2}}+\frac{1}{2}\left\{\cos \left(\frac{\pi}{4}-x\right) x^{-\frac{1}{2}}+\frac{1}{2} \int x^{-\frac{3}{2}} \cos \left(\frac{\pi}{4}-x\right) d x\right\} \\
& (3)=-\sin \left(\frac{\pi}{4}-x\right) x^{\frac{1}{2}}+\frac{1}{2} \cos \left(\frac{\pi}{4}-x\right) x^{-\frac{1}{2}}+\frac{1}{4} \int x^{-\frac{3}{2}} \cos \left(\frac{\pi}{4}-x\right) d x
\end{aligned}
$$

But in the last term of the above equation we have, 

by the lav of the mean,

$$
\begin{aligned}
\int_{a}^{\lambda} x^{-\frac{3}{2}} \cos \left(\frac{\pi}{4}-x\right) d x & =\cos \left(\frac{\pi}{4}-\bar{x}\right) \int x^{-\frac{3}{2}} d x \\
& =-2 \cos \left(\frac{\pi}{4}-\bar{x}\right) x^{-\frac{1}{2}},
\end{aligned}
$$

where $\bar{x}$ lies between $a$ and $\lambda$. This term is of the order $x^{-\frac{1}{2}}$ and can be included in the $R$ of equation (2). The same is true of the second term of equation (3).

Hence, we w1ll now have:

$$
\lambda J_{1}(\lambda)-a J_{1}(a)=\frac{2}{\sqrt{2 \pi}}\left\{-\left.\sin \left(\frac{\pi}{4}-x\right) x^{\frac{1}{2}}\right|_{a} ^{\lambda}+R_{1}\right\}
$$

where $R_{1}-\left[-\theta 4 \sqrt{\frac{2}{\pi}} g^{2} e^{-1} x^{-\frac{1}{2}}+\frac{1}{2} \cos \left(\frac{\pi}{4}-x\right) x^{-\frac{1}{2}}-\left.\frac{1}{2} \cos \left(\frac{\pi}{4}-\bar{x}\right) x^{-\frac{1}{2}}\right|_{a} ^{\lambda}\right.$ Then

$$
\lambda J_{1}(\lambda)=a J_{1}(a)+\frac{2}{\sqrt{2 \pi}}\left\{\sin \left(\lambda-\frac{\pi}{4}\right) \lambda^{\frac{1}{2}}-\sin \left(a-\frac{\pi}{4}\right) a^{\frac{1}{2}}\right\}+R_{1}
$$

and

$$
\begin{aligned}
\lambda J_{1}(\lambda)-\frac{2}{\sqrt{2 \pi}} \sin \left(\lambda-\frac{\pi}{4} \lambda^{\frac{1}{2}}-R_{1}(\lambda)\right. & \\
& =a J_{1}(a)-\frac{2}{\sqrt{2 \pi}} \sin \left(a-\frac{\pi}{4}\right) a^{\frac{1}{2}}-R_{1}(a) .
\end{aligned}
$$

Hence if a is a constant

$$
\lambda J_{1}(\lambda)-\frac{2}{\sqrt{2 \pi}} \sin \left(\lambda-\frac{\pi}{4} \lambda^{\frac{1}{2}}-R_{1}(\lambda)=\mathrm{K},\right. \text { a constant. }
$$

We will now prove that this constant is zero.

It is a well known fact that in any range $2 \pi$ of the 

variable, $J_{l}(\lambda)$ has at least one root and the same is true of $\lambda J_{1}(\lambda)$. The function $\frac{2}{\sqrt{2 \pi}} \sin \left(\lambda-\frac{\pi}{4}\right) \lambda^{\frac{1}{2}}$ also has roots lying at intervals of $\pi$ apart. Hence the two curves must cross. Suppose they cross at $\lambda=\lambda^{*}$. For such a value, $\mathrm{R}_{1}\left(\lambda^{*}\right)=-\mathrm{k}$. But such an interval can be found so that $\lambda^{*}$ is as big as you please and since $R_{1}(\lambda)$ is of order $\frac{1}{\sqrt{\lambda}}, R_{1}\left(\lambda^{*}\right)$ can be made as small as we please.

Hence $R_{l}\left(\lambda^{*}\right)=0$ and consequently $K=0$.

Thus we have:

$$
\lambda J_{1}(\lambda)=\frac{2}{\sqrt{2 \pi}} \sin \left(\lambda-\frac{\pi}{4} \lambda^{\frac{1}{2}}+R_{1}(\lambda) \text {, where }\left|R_{1}(\lambda)\right|<\frac{c}{\sqrt{\lambda}}\right.
$$

C being a constant.

Now the maxima of this function are not less in absolute value than $\frac{2}{\sqrt{2 \pi}} \sqrt{\mu_{i}}-\frac{c}{\sqrt{\mu_{i}}}$, where $\mu_{i}$ are the roots of $J_{0}(x)=0$. The general term of the series which we are investigating can be written:

$$
\frac{1}{\mu_{i}^{m-2}\left(\mu_{i}{ }^{J}{ }_{1}\left(\mu_{i}\right)\right)^{2}}
$$

This now becomes:

$$
\frac{1}{\mu_{i}^{m-2}\left(\mu_{i} J_{1}\left(\mu_{i}\right)\right)^{2}}<\frac{1}{\mu_{i}^{m-2} \frac{4}{2 \pi} \mu_{i}-\frac{4 C}{\sqrt{2 \pi}}-\frac{C^{2}}{\mu_{i}}}<\frac{1}{\mu_{i}^{m-2} \frac{1}{\left(\frac{4}{2 \pi} \mu_{i}-\frac{4 C}{\sqrt{2 \pi}}\right.}}
$$

Or if $\frac{2 \mu_{i}>\frac{4 C}{2 \pi}}{\sqrt{2 \cdot \pi}}$ our general term is less than $\frac{\pi}{\mu_{i}^{m-1}}$

But no roots of $J_{0}(x)=0$ are closer spaced than $\frac{\pi}{2}$. Hence $\mu_{i}>\pi y / 2$ and the $i^{\text {th }}$ term of our series becomes less than $\frac{\pi}{\left(\frac{\pi i}{2}\right)^{m-1}}$ or $\frac{2^{m-1}}{\pi^{m-2}} \frac{1}{i^{m-1}}, i=1,2,3, \ldots$

Hence our series converges and converges at least as 

fast as $\mathbb{N} \sum_{n=1}^{\infty} \frac{1}{n-1}$, where $N$ is a constant. It is of course clear that we will have to choose $m>2$. 







This thesispis hever to jeave this room Neither if night.

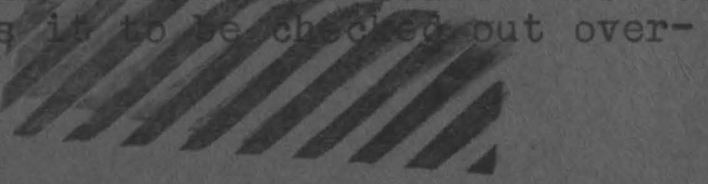


